\title{
Paediatric respiratory infections
}

\author{
Mark L. Everard
}

CrossMark

Affiliation: School of Paediatrics and Child Health, University of Western Australia, Princess Margaret Hospital, Subiaco, Australia.

Correspondence: Mark L. Everard, School of Paediatrics and Child Health, University of Western Australia, Princess Margaret Hospital, Roberts Road, Subiaco, Western Australia, 6008, Australia.

E-mail: mark.everardduwa.edu.au

ABSTRACT Pulmonary infections remain a major cause of infant and child mortality worldwide and are responsible for a substantial burden of morbidity. During the 2015 European Respiratory Society International Congress in Amsterdam, some of the main findings from peer-reviewed articles addressing this topic that were published in the preceding 12 months were reviewed in a Paediatric Clinical Year in Review session. The following article highlights some of the insights provided by these articles into the complex interactions of the human host with the extensive and dynamic populations of microorganisms that call an individual "home".

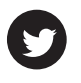

@ERSpublications

A review of publications from the past year addressing acute life-threatening and chronic respiratory infections http://ow.ly/W4ahI

\section{Introduction}

Much of our approach to infectious diseases over the past 125 years has been shaped by Koch's postulates formulated in the later part of the 19th century. This approach was invaluable in identifying organisms responsible for life-threatening diseases such as pneumococcal pneumonia, meningococcal meningitis, influenza and tuberculosis. In turn, these insights led to the development of directed therapy in the form of antibiotics for bacterial organisms and preventative strategies using immunisation for many of the more aggressive organisms.

However, as we move from an era in which death in childhood from infectious respiratory disease was common to one in which mortality is now very low, at least in developed countries, attention is turning increasingly to the impact of more chronic infectious diseases and mechanisms underlying acute but non-life-threatening infectious diseases. The recognition of the importance of biofilms in chronic diseases such as persistent bacterial bronchitis, chronic otitis media and chronic sinusitis, the bacterial-viral interactions associated with something as simple as a "head cold" and the impact of complex microbiota in the gut and airways on a variety of chronic diseases have highlighted the limitations inherent in the traditional one-organism/one-disease model proposed by Koch.

The huge advances observed in sequencing and other technologies over the past two decades are starting to have a significant impact on our understanding of the role of the human body as an environmental niche colonised by huge numbers of microorganisms that can have a range of effects on the health of the host and which in turn are influenced by the host response and other environmental determinants. This technology is also now being used to improve our understanding of the epidemiology of outbreaks of infectious disease and the evolution of organisms in response to environmental challenges such as availability of nutritional factors, antibiotics and the influx of novel organisms acquired from other humans or the environment.

Received: Nov 072015 | Accepted after revision: Dec 152015

Conflict of interest: None declared.

Provenance: Submitted article, peer reviewed.

Copyright CERS 2016. ERR articles are open access and distributed under the terms of the Creative Commons Attribution Non-Commercial Licence 4.0. 


\section{Contrasting impacts of acute respiratory infections in developing and developed countries}

A comparison of data relating to infant and child mortality from rural Kenya [1] with data from North America looking at hospitalisation with "radiologically proven pneumonia" [2] highlights the difference in challenges facing the different healthcare systems. In rural Kenya, infant and childhood mortality (60 and 20 per 1000 live births, respectively) remains very high when compared with that observed in developed countries, even though infant mortality almost halved over the 8 years (2003-2010) of the study. Most of this reduction was attributable to reducing death due to respiratory infections, and while respiratory infections were the commonest cause of death over the whole period, by 2010 mortality among infants due to malaria had overtaken respiratory infections. In contrast, a study from the USA noted that the rate of admission to hospital with radiologically proven pneumonia was only 6.2 per 1000 among infants and 1.6 per 1000 children. Out of 2358 children admitted to hospital there were only three deaths. Curiously, in this study 33\% of subjects had been labelled as having "asthma" or "reactive airways disease" [3]. Such low levels of mortality resulting from respiratory infections in developed nations is probably due to a combination of factors such as vaccination, ready access to antibiotics, nutrition, low exposure to biofuel combustion and improved sanitation.

\section{Diagnosis of pneumonia and acute lower respiratory tract infections}

A systematic review of a large number of papers addressing the utility of a wide range of signs in accurately identifying infants with pneumonia confirmed the well-established finding that no one sign or indeed combination of signs reliably identified infants with pneumonia [4]. As noted in an editorial [5], the World Health Organization (WHO) approach based on respiratory rate greatly overestimates the incidence of pneumonia, but was designed to ensure the early use of antibiotics in resource-poor countries to try and impact on the high levels of mortality. The editorial goes on to emphasise the impact of vaccination and the need for more precise point-of-care diagnostics, which may be based on metabolomics approaches [6]. The results of the systematic review seriously question the validity of guidelines such as the British Thoracic Society's paediatric pneumonia guidelines, which essentially ignore data showing the poor specificity of clinical assessment.

\section{Identification of pathogens and relationship to disease and disease severity}

In the study by JAIN et al. [2], bacterial pathogens were identified in a minority of cases, with viruses, particularly respiratory syncytial virus (RSV) in the younger subjects and rhinovirus in older children, being identified in the majority. More than one virus or a virus and bacteria were identified in 15-30\%, depending on age. Inevitably it is tempting to ascribe a causal relationship when a "respiratory pathogen" is identified in the airways of subjects with an acute illness. It has long been known that potential bacterial pathogens such as Streptococcus pneumoniae, Moraxella catarrhalis and Haemophilus influenzae species are commonly identified in the nose and nasopharynx of apparently asymptomatic subjects. A number of studies have confirmed that the same is true for viruses, such that some "respiratory" viruses are more likely to be identified in asymptomatic infants and children than symptomatic subjects. PRINCIPI et al. [7] prospectively undertook weekly sampling of the upper airways of 88 infants. 326 samples were positive for human rhinovirus using PCR, of which 209 (64\%) were from asymptomatic infants. The likelihood of symptoms in conjunction with a positive swab (upper respiratory tract $19 \%$ and/or lower respiratory tract $14 \%$ ) was not influenced significantly by the type of rhinovirus (A, B or C). None of the infants were hospitalised. In a similar study of a panel of viruses by CHONMAITREe et al. [8] a virus was identified in 795 (39\%) of samples, with two or more viruses in 228 of these. Predictably, rhinovirus was the most commonly identified virus. A virus was identified in $28 \%$ of asymptomatic infants, accounting for $53 \%$ of all positive samples. A further 75 (51\%) samples were positive in asymptomatic subjects who had experienced upper respiratory symptoms in the previous week. The authors calculated that the odds ratio of a positive viral identification in a symptomatic subject (again all relatively mild community infections), as opposed to an asymptomatic infant with RSV, was 11.7. RHEDIN et al. [9] found that the identification of RSV, human metapneumovirus (hMPV), influenza and adenovirus was significantly higher in cases of community-acquired pneumonia than in healthy controls, but this was not the case for rhinovirus and other viruses such as bocavirus. A broader systematic review of published literature in relation to acute lower respiratory tract infections by SHI et al. [10] essentially confirmed these findings, reporting strong evidence for causal attribution of RSV (OR 9.8), influenza (OR 5.0), parainfluenza (OR 3.4) and hMPV (OR 3.8).

Pertinent to the issue of symptomatic and asymptomatic infections was the study of Henriquez et al. [11], who found a close correlation between levels of interleukin (IL)-8 and numbers of neutrophils in the upper airway with duration and severity of respiratory symptoms, confirming earlier studies emphasising the importance of IL-8, neutrophils and their products in the causation of symptoms induced by "respiratory" viruses $[12,13]$. It has long been recognised that there are interactions between viruses and 
bacteria in the airways. Staphylococcal pneumonia following influenza infection was responsible for a large proportion of the deaths in the 1919 pandemic; chickenpox predisposes to severe group A streptococcal pneumonia and infection at other sites; and, more recently, the role of viruses in altering the behaviour of biofilms during exacerbations has been the subject of research. Even the severity of a simple "cold" appears to be driven in large part by the load of potential pathogenic bacteria in the nose and nasopharynx rather than the simple presence of a respiratory virus [14], which, as noted earlier may be totally asymptomatic for most viruses. Studies using the conjugate pneumococcal vaccine in South Africa found reductions in admissions with apparent "viral-induced lower respiratory tract infection", further indicating the influence of bacteria on the impact of viruses [15]. A study by NGUYEN et al. [16] in a rodent model provided some insight into this, showing that animals infected with $S$. pneumoniae and inoculated with RSV 3 days later had significantly higher levels of virus in their upper airways 5 days later than in those mock-infected with $S$. pneumoniae. Increasingly we are recognising that a microorganism must not only interact with the host and its defences, but must also compete and/or collaborate with other microorganisms. As noted by Rodrigues et al. [14], the Sino-Nasal Outcome Test score of a cold will be driven by host responses largely mediated by neutrophils (whose products not only drive symptoms but provide the colouration of the nasal discharge), and the magnitude of this response is likely to be driven by type of virus, viral load and presence of potentially pathogenic bacteria.

\section{Microbiome}

As a noted earlier, the ability to undertake detailed genetic sequencing is providing further insights into the resident populations that appear to see any niche within the body as a potential home. Studies using $16 \mathrm{~S}$ ribosomal RNA sequencing to identify bacterial populations have indicated the huge numbers and diversity of organisms in the mouth and nose and the large difference between the two. Populations are dynamic and can change over short periods of time. These populations are both susceptible to disruption with interventions such as acquisition of a virus or exposure to antibiotics, and relatively resilient, generally reverting to pre-disruption patterns relatively quickly in many cases. While there is still some uncertainty about the existence of a pulmonary microbiome, well-constructed studies suggest that the lower airway is probably colonised even in healthy individuals. Two recent studies suggest that micro-aspiration rather inhalation is the primary route taken by organisms establishing themselves in the lower airway $[17,18]$. Due to the extreme sensitivity of these techniques it is critical that such studies are performed with great care, both in terms of obtaining the samples and in quality control when processing them, as exemplified by SALTER et al.'s [19] salutary paper, which provides examples of apparently significant results being entirely due to contamination, either in the sample or in the reagents used to process the samples. Challenges such as these and the dynamic and constantly changing populations mean that the interpretation of data must to be undertaken with caution.

\section{Imaging}

A group from Perth (Australia) and their collaborators in the Netherlands have published a more sensitive computed tomography (CT) scoring system for use in cystic fibrosis, in large part aimed at improving the utility of images when used as an end-point in clinical trials [20]. Perhaps the most noteworthy feature is the extremely low doses of radiation required, with optimal tailoring of scanners for the purpose largely removing the concerns raised in the past about use of CT to monitor disease progress in cystic fibrosis. Indeed, the data suggest that, rather than the use of this approach being unethical, it is unethical not to ensure that the imaging is optimised to ensure that such low levels of radiation are used. A study by CIET et al. [21] found magnetic resonance imaging to be valuable in pulmonary imaging in cystic fibrosis, but, at least in their hands, somewhat less sensitive than CT imaging. However, they did propose to explore its utility further.

\section{Acute bronchiolitis}

A number of studies failed to support the conclusions of a Cochrane review that hypertonic saline may be useful in the management of acute bronchiolitis, adding a further intervention to the long list of ineffective interventions [22, 23]. A large study by Cunningham et al. [24] highlighted the impact of administering arbitrary levels of oxygen on duration of hospitalisation in a well-designed randomised double-blind trial involving $>500$ infants. Those in whom oxygen therapy was discontinued when their saturations in room air remained $>90 \%$ were discharged significantly more rapidly without any adverse events. However, this study focused on weaning from oxygen and the results should not be interpreted as indicating that it is safe to discharge infants with a saturation $>90 \%$ at an early time point in their illness.

\section{Maternal immunisation}

In order to address the rising mortality noted in very young infants contracting pertussis before they are immunised, a number of countries have recently introduced routine immunisation of mothers during 
pregnancy. A number of studies have reviewed the impact of this change in policy, with studies from the UK [25] and USA [26] indicating that the strategy appears to be safe and effective, although a very recent study has highlighted the impact of maternal immunisation on the response to postnatal pertussis immunisation and other vaccines in the routine immunisation programme that are conjugated to the diphtheria toxin variant [27]. Other groups report a reduction of morbidity in the mothers and their infants when mothers are immunised with influenza vaccine during pregnancy [28]. The effect is not as great as that observed with pertussis and the authors do note the challenges in interpreting the data and providing recommendations, given seasonal and year-to-year variations in disease severity and incidence. The same approach has been advocated for some time by a number of groups working on RSV vaccines, and proceedings of a WHO meeting about vaccines for this particularly troublesome respiratory virus have been published [29].

\section{Sequencing as a clinical and research tool}

As noted above, sequencing and omics platforms are impinging on research in the microbiome field and may start to play a central role in diagnostics as metabolomics comes of age. Other examples of powerful gene-sequencing techniques introduced into the clinical space include a report of whole-exome sequencing being used to increase the ability to identify the underlying genetic defect in those with primary ciliary dyskinesia [30] and a study in which whole-genome sequencing results indicated that the risk of within-clinic transmission of Mycobacterium abscessuss appears to be very low (although a sibling pair carried genetically identical organisms) [31]. Sequencing also provided further evidence that RSV is subject to relatively few evolutionary pressures, supporting the suggestion that it has adopted an effective strategy for success that obviates the need for major antigenic shifts or the evolution of multiple subtypes [32].

In summary, publications this past year have again highlighted the dramatic difference between respiratory mortality among infants and young children in high- and low-resource countries. A focus on the traditional major respiratory pathogens will continue as improved approaches to prevention are sought, but there is also an increasing emphasis on the role of host and microbiome interactions in the causation and/or perpetuation of chronic disease. This has been facilitated by the rapid developments in sequencing technology over the past decade and which in time are likely to lead to novel approaches to the prevention and management of both acute and chronic respiratory diseases.

\section{References}

1 Desai M, Buff AM, Khagayi S, et al. Age-specific malaria mortality rates in the KEMRI/CDC health and demographic surveillance system in western Kenya, 2003-2010. PLoS One 2014; 9: e106197.

2 Jain S, Finelli L, CDC EPIC Study Team. Community-acquired pneumonia among U.S. Children. N Engl J Med 2015; 372: 2167-2168.

3 George M, Ahmad SQ, Wadowski S, et al. Community-acquired pneumonia among U.S. Children. N Engl J Med 2015; 372: 2166-2167.

4 Rambaud-Althaus C, Althaus F, Genton B, et al. Clinical features for diagnosis of pneumonia in children younger than 5 years: a systematic review and meta-analysis. Lancet Infect Dis 2015; 15: 439-450.

5 Qazi S, Were W. Improving diagnosis of childhood pneumonia. Lancet Infect Dis 2015; 15: 372-373.

6 Joensen O, Paff T, Haarman EG, et al. Exhaled breath analysis using electronic nose in cystic fibrosis and primary ciliary dyskinesia patients with chronic pulmonary infections. PLoS One 2014; 9: el15584.

7 Principi N, Zampiero A, Gambino M, et al. Prospective evaluation of rhinovirus infection in healthy young children. J Clin Virol 2015; 66: 83-89.

8 Chonmaitree T, Alvarez-Fernandez P, Jennings K, et al. Symptomatic and asymptomatic respiratory viral infections in the first year of life: association with acute otitis media development. Clin Infect Dis 2015; 60: 1-9.

9 Rhedin S, Lindstrand A, Hjelmgren A, et al. Respiratory viruses associated with community-acquired pneumonia in children: matched case-control study. Thorax 2015; 70: 847-853.

10 Shi T, McLean K, Campbell H, et al. Aetiological role of common respiratory viruses in acute lower respiratory infections in children under five years: a systematic review and meta-analysis. J Glob Health 2015; 5: 010408.

11 Henriquez KM, Hayney MS, Xie Y, et al. Association of interleukin-8 and neutrophils with nasal symptom severity during acute respiratory infection. J Med Virol 2015; 87: 330-337.

12 Everard ML, Swarbrick A, Wrightham M, et al. Analysis of cells obtained by bronchial lavage of infants with respiratory syncytial virus infection. Arch Dis Child 1994; 71: 428-432.

13 Abu-Harb M, Bell F, Finn A, et al. IL-8 and neutrophil elastase levels in the respiratory tract of infants with RSV bronchiolitis. Eur Respir J 1999; 14: 139-143.

14 Rodrigues F, Foster D, Nicoli E, et al. Relationships between rhinitis symptoms, respiratory viral infections and nasopharyngeal colonization with Streptococcus pneumoniae, Haemophilus influenzae and Staphylococcus aureus in children attending daycare. Pediatr Infect Dis J 2013; 32: 227-232.

15 Madhi SA, Klugman KP, Vaccine Trialist Group. A role for Streptococcus pneumoniae in virus-associated pneumonia. Nat Med 2004; 10: 811-813.

16 Nguyen DT, Louwen R, Elberse K, et al. Streptococcus pneumoniae enhances human respiratory syncytial virus infection in vitro and in vivo. PLoS One 2015; 10: $\mathrm{e} 0127098$.

17 Bassis CM, Erb-Downward JR, Dickson RP, et al. Analysis of the upper respiratory tract microbiotas as the source of the lung and gastric microbiotas in healthy individuals. MBio 2015; 6: e00037.

18 Boutin S, Graeber SY, Weitnauer M, et al. Comparison of microbiomes from different niches of upper and lower airways in children and adolescents with cystic fibrosis. PLoS One 2015; 10: e0116029. 
19 Salter SJ, Cox MJ, Turek EM, et al. Reagent and laboratory contamination can critically impact sequence-based microbiome analyses. BMC Biol 2014; 12: 87.

20 Rosenow T, Oudraad MC, Murray CP, et al. PRAGMA-CF. A quantitative structural lung disease computed tomography outcome in young children with cystic fibrosis. Am J Respir Crit Care Med 2015; 191: 1158-1165.

21 Ciet P, Serra G, Bertolo S, et al. Assessment of CF lung disease using motion corrected PROPELLER MRI: a comparison with CT. Eur Radiol 2015 [in press; DOI: 10.1007/s00330-015-3850-9].

22 Everard ML, Hind D, Ugonna K, et al. SABRE: a multicentre randomised control trial of nebulised hypertonic saline in infants hospitalised with acute bronchiolitis. Thorax 2014; 69: 1105-1112.

23 Teunissen J, Hochs AH, Vaessen-Verberne A, et al. The effect of 3\% and 6\% hypertonic saline in viral bronchiolitis: a randomised controlled trial. Eur Respir J 2014; 44: 913-921.

24 Cunningham S, Rodriguez A, Adams $\mathrm{T}$, et al. Oxygen saturation targets in infants with bronchiolitis (BIDS): a double-blind, randomised, equivalence trial. Lancet 2015; 386: 1041-1048.

25 Amirthalingam G, Andrews N, Campbell $\mathrm{H}$, et al. Effectiveness of maternal pertussis vaccination in England: an observational study. Lancet 2014; 384: 1521-1528.

26 Moro PL, McNeil MM, Sukumaran L, et al. The Centers for Disease Control and Prevention's public health response to monitoring Tdap safety in pregnant women in the United States. Hum Vaccin Immunother 2015; 11: 2872-2879.

27 Ladhani SN, Andrews NJ, Southern J, et al. Antibody responses after primary immunization in infants born to women receiving a pertussis-containing vaccine during pregnancy: single arm observational study with a historical comparator. Clin Infect Dis 2015; 61: 1637-1644.

28 Omer SB, Richards JL, Madhi SA, et al. Three randomized trials of maternal influenza immunization in Mali, Nepal, and South Africa: methods and expectations. Vaccine 2015; 33: 3801-3812.

29 Modjarrad K, Giersing B, Kaslow DC, et al. WHO consultation on respiratory syncytial virus vaccine development report from a World Health Organization meeting held on 23-24 March 2015. Vaccine 2016; 34: 190-197.

30 Marshall CR, Scherer SW, Zariwala MA, et al. Whole-exome sequencing and targeted copy number analysis in primary ciliary dyskinesia. G3 2015; 5: 1775-1781.

31 Harris KA, Underwood A, Kenna DT, et al. Whole-genome sequencing and epidemiological analysis do not provide evidence for cross-transmission of Mycobacterium abscessus in a cohort of pediatric cystic fibrosis patients. Clin Infect Dis 2015; 60: 1007-1016.

32 Bose ME, He J, Shrivastava S, et al. Sequencing and analysis of globally obtained human respiratory syncytial virus A and B genomes. PLoS One 2015; 10: e0120098. 